\title{
Digital Image Correlation of Heterogeneous Deformation in Polycrystalline Material with Electron Backscatter Diffraction
}

\author{
Javier Esquivel $^{1}$, Michael D. Sangid ${ }^{1}$ \\ 1. Purdue University, School of Aeronautics and Astronautics, West Lafayette, IN, USA.
}

This work establishes the ability to conduct digital image correlation (DIC) investigations at length scales that elucidate slip system activation and quantification as the primary deformation mechanism in polycrystalline materials. DIC allows for a computational method of strain field measurements using multiple images to track random speckle patterns on material surfaces. Self-assembling gold nanoparticles provide sub-micron resolution speckle patterns to study microstructure influences on deformation using scanning electron microscopy (SEM). The complex microstructures in aerospace grade aluminum give rise to varied deformation fields, which can be studied using electron backscatter diffraction (EBSD). Specimen preparation techniques, speckle patterns, and image correlation analysis are discussed. Experimental identification of strains at grain level can help validate computational crystal plasticity finite element models, which in turn provide better predictive computational models.

Component failure is a result of deformation accumulating in small regions within a part. In fact, strain localization is a precursor to material failure. In this research, the fundamentals of strain localization are enabled through the set-up of a unique experimental analysis. The microstructure attributes, especially grain boundaries (GBs), are responsible for heterogeneous deformation. As defects within the microstructure localizes deformation and leads to incompatible strain. DIC represents a powerful tool when combined with characterization of the microstructure, to assess the heterogeneous deformation, damage mechanisms, or fatigue behavior [1-3].

In the following research, a specimen is constructed (Fig. 1a), polished, and fiducial marks are placed on the specimen in the form of microhardness indents to establish a spatial reference for microanalysis (Fig 1b). The spatial crystallographic orientations are measured via EBSD and overlaid on the specimen according to the fiducial marks in Fig. 1c. A gold nanoparticle speckle pattern is applied to the material as shown in Fig. 2 according to the novel procedure developed by Kammers and Daly [4]. For this technique, the sample is hydroxylated to increase the oxide and hydroxide groups via a basic solution bath. The specimen is incubated in silane for 1 day and then a colloidal gold solution for a week to attach silane linker molecules and gold nanoparticles to the functionalized silane molecules, respectively. Reference images of the speckle pattern are taken at 5000x in the SEM via a grid of 6x5 images $(24.79 \times 18.6 \mu \mathrm{m}$ per image) within the $100 \mu \mathrm{m}$ by $100 \mu \mathrm{m}$ fiducial markers, as shown in Fig. $3 \mathrm{a}$. The Al specimen was loaded and unloaded exhibiting a $1.4 \%$ residual strain. Images of the deformed speckle pattern were acquired and the deformation was correlated using VIC-2D software.

The strain fields of DIC are superimposed on the crystallographic orientation (Fig. 3b), thus elucidating the GBs in the material. Further, through the crystallographic orientations, the slip planes and GBs are indexed [1]. The results allowed for quantification of local plastic deformation relative to the material's microstructure, in the form of slip bands and represented resolution necessary to display slip system activation. The results are important, as localization of deformation at the slip band level is crucial to failure processes, such as fatigue. Further, the results display spacing in between slip bands, the amount of deformation accommodated by each slip band, and the discontinuous nature of slip. 
[1] W. Abuziad, M.D. Sangid, J. Carroll, H. Sehitoglu and J. Lambros, Journal of the Mechanics and Physics of Solids 60 (2012), p. 1201-1220.

[2] A.D. Kammers and S. Daly, Experimental Mechanics 53 (2013), p. 1743-1761.

[3] F. Di Gioacchino and J. Quinta da Fonseca, Experimental Mechanics 53 (2013), p. 743-754.

[4] A.D. Kammers and S. Daly, Experimental Mechanics 53 (2013), p. 1333-1341.

[5] The authors gratefully acknowledge German A. Parada for initial set-up of the speckle pattern procedure during undergraduate research at Purdue. The authors graciously acknowledge support for this work from Rolls-Royce Corporation and the National Science Foundation, CMMI-1334664. Prof. Daly is thanked for providing stimulating discussions and suggestions for the SEM-DIC methodology.
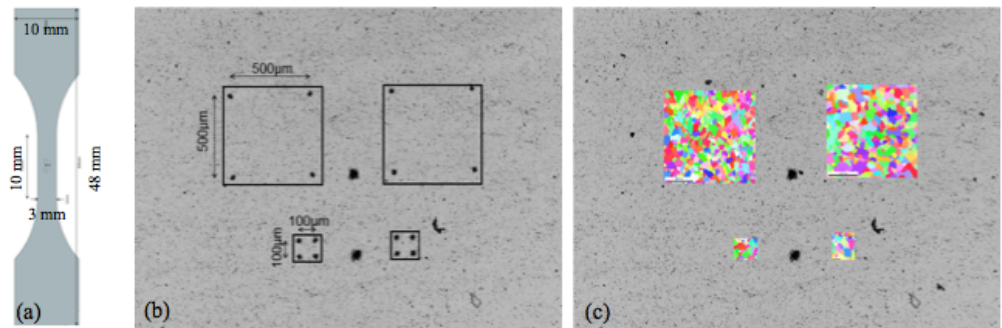

Figure 1. (a) Al specimen. (b) Microhardness indentations displaying fiducial marks to elucidate regions of interest. (c) Superimposed information of crystallographic orientations via EBSD.

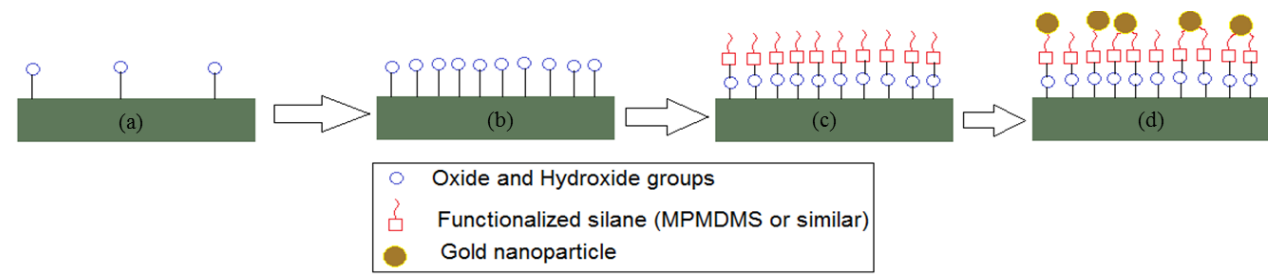

Figure 2. Gold nanoparticle surface layer preparation (a) Standard oxide and hydroxide groups. (b) Increased oxide and hydroxide groups after basic solution bath. (c) Silane linker molecules after MPMDMS bath. (d) Gold nanoparticles attach to functionalized silane molecules.

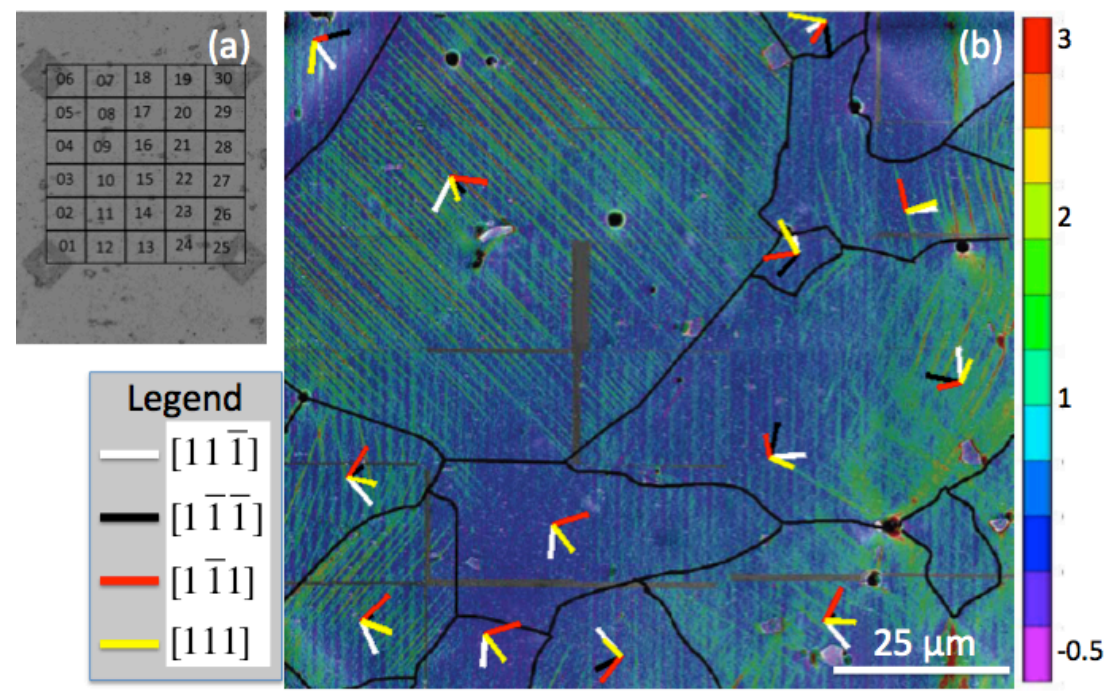

Figure 3. (a) $100 \mu \mathrm{m}$ by $100 \mu \mathrm{m}$ region of interest -30 images acquired at 5000x. (b) Concurrent measurements of material deformation via DIC in \% axial strain and crystallographic orientations via EBSD to measure $1.4 \%$ residual strain. 